\title{
Leibliches Beten bei Etty Hillesum
}

Pierre Bühler

\section{Zum Einstieg: ein Wittgenstein-Zitat}

In einer Notiz Wittgensteins von 1946 heisst es: »Ich kann nicht niederknien, zu beten, weil gleichsam meine Knie steif sind. Ich fürchte mich vor der Auflösung (vor meiner Auflösung), wenn ich weich würde. « ${ }^{1}$ Fünf Jahre früher, im unter deutscher Besatzung stehenden Amsterdam schreibt eine junge Jüdin namens Etty Hillesum im November 1941 in ihrem Tagebuch, sie würde gerne eine Novelle zum Thema "Das Mädchen, das nicht knien konnte " schreiben. ${ }^{2}$ Einige Seiten später, am darauffolgenden Tag, heisst es dann: „Den Vorgang in mir, wie das Mädchen knien lernte, möchte ich in all seinen Abstufungen schildern. "Deshalb scheint sich nun das Thema der geplanten, aber anscheinend nie geschriebenen Novelle bereits etwas verschoben zu haben: „Das Mädchen, das nicht knien konnte und es dann doch lernte auf einer rauen Kokosmatte in einem unordentlichen Badezimmer. $\star^{3}$

Vermutlich weiss man nicht, ob Wittgenstein das Niederknien doch noch erlernt hat. Es ist eher unwahrscheinlich. Die Furcht vor der Auflösung, vor dem Weichwerden, scheint zu gross zu sein. Interessant ist dabei in der kurzen Notiz der Kontrast zwischen "steif" und »weich«. Was als ein Gebrechen, ein leibliches Defizit erscheinen könnte, die steifen Knie, wird anscheinend als willkommener Schutz vor der Gefahr des Erweichens erachtet.

Bei Etty Hillesum hingegen lässt sich in den Tagebüchern beobachten, wie sie zu einer regelmässig niederknienden Beterin wird, und zwar nicht etwa in der Synagoge, sondern an alltäglichen Orten wie ihrer Kammer oder eben im unordentlichen Badezimmer! Was

1 Ludwig Wittgenstein, Vermischte Bemerkungen, Frankfurt a.M. 1977, 109.

2 Etty Hillesum, Das denkende Herz der Baracke. Die Tagebücher 1941-1943, Freiburg/Basel/Wien, Herder, 2014, 102 [21.11.1941]. Diese deutsche Übersetzung bietet leider nur eine Auswahl aus den Tagebüchern und Briefen von Etty Hillesum; deshalb übersetze ich einige Stellen aus der französischen Gesamtausgabe: Les écrits d'Etty Hillesum. Journaux et lettres, 1941-1943. Édités sous la direction de Klaas A.D. Smelik, Paris, Éditions du Seuil, 2008.

3 Das denkende Herz der Baracke, beide Zitate auf S. 106 [22.11.1941]. 
hat diese leibliche Geste des Niederkniens zu bedeuten? Diese Frage ist umso interessanter, als die Geste des Niederkniens eigentlich nicht einer jüdischen Gebetshaltung entspricht, sondern bei Etty Hillesum, weil sie aus einer relativ assimilierten jüdischen Familie stammt, anscheinend aus der christlich geprägten Umwelt übernommen wurde. Doch auch als sie dann unter dem Druck der Judenverfolgung und -deportation im Laufe von 1942 immer stärker ihre jüdischen Wurzeln wiederentdeckte, blieb sie weiterhin bei dieser Gebetshaltung. Im Niederknien kam für sie etwas Entscheidendes zum Ausdruck, und das soll im Folgenden kurz aufgespürt werden.

Doch bevor wir hier fortfahren, soll zunächst für diejenigen, welche sie vielleicht nicht kennen, knapp geschildert werden, wer Etty Hillesum war, deren 100. Geburtstag dieses Jahr gefeiert wird.

\section{Wer ist Etty Hillesum?}

Etty Hillesum wurde am 15. Januar 1914 in Middelburg, Niederlanden, geboren, als erstes von drei Kindern eines jüdischen Ehepaars. Ihr Vater war Gymnasiallehrer für klassische Sprachen und ihre Mutter kam aus Russland, wo sie einem Pogrom entfliehen konnte, und gab zunächst Russisch-Privatstunden. Die Familie war relativ assimiliert, doch gewannen die jüdischen Wurzeln für Etty und ihre Familie in der Zeit der Besetzung der Niederlanden durch Deutschland an Bedeutung. Nach Abschluss des Gymnasiums in Deventer kam sie 1932 nach Amsterdam, wo sie Rechtswissenschaft studierte. Danach lernte sie slawische Sprachen, las Dostojewski und gab Russischstunden. Im Frühling 1941 begegnete sie einem deutschen Juden, der in Amsterdam im Exil lebte, Julius Spier. Er wurde für Etty Hillesum ein enger Freund und zugleich ein geistlicher Lehrer. Unter seiner Anleitung las sie Jung, aber auch die Bibel, Augustin, Meister Eckhart, und, wie auch früher schon, viel Rilke. Aber sie begann auch ein Tagebuch zu schreiben. Zehn Hefte (von März 1941 bis Oktober 1942) sind erhalten geblieben. Sie geben Einblick in die geistliche Entwicklung einer jungen Frau im Zeichen der immer schlimmer werdenden Unterdrückung ihres Volkes, mit dem sie sich mehr und mehr verbunden fühlt. Als die ersten Deportationen der niederländischen Juden nach Auschwitz und anderen Konzentrationslagern einsetzten, liess sie sich als Helferin anstellen, zuerst in Amsterdam, und dann im Transitlager Westerbork. In ergreifenden Briefen an Freunde hat sie die alltägliche Not im Transitlager beschrieben. Nachdem die Helfer ihren Sonderstatus verloren hatten, 
wurde sie selbst im September 1943, zusammen mit ihren Eltern und einem Bruder, nach Auschwitz deportiert. Vom Roten Kreuz wird der 30. November 1943 als Todesdatum angegeben. Ihr letztes Zeugnis ist eine Postkarte, die sie aus dem Zug warf und die man später auf den Gleisen fand.

\section{"Eine in meinen Leib eingegossene Geste»}

Früh bereits in den Tagebüchern taucht die Ahnung auf, dass die Möglichkeit des Betens bestünde, obschon diese Möglichkeit zunächst nicht zur Wirklichkeit wird. In einem Eintrag vom 16. März 1941 notiert sie, wie sie auf der Terrasse, in der Frühlingssonne, eine neue Art Empfindung erlebt, in der sie nicht mehr versucht, alles $\mathrm{zu}$ beherrschen und $\mathrm{zu}$ durchdringen, sondern sich von Kräften füllen lässt, die sie glücklich macht. Diese Erfahrung nimmt plötzlich leibliche Gestalt an: "während ich dort an der Sonne sass, habe ich unbewusst den Kopf geneigt, wie um dieses neue Lebensgefühl noch besser zu empfinden". Dieses unbewusste Neigen des Kopfes scheint die Assoziation des Betens zu stiften, denn im darauf folgenden Satz heisst es: „Und so spürte ich plötzlich tief in mir wie ein Mensch tosend auf die Knie fallen und sich dann beruhigen kann, das Gesicht in seinen gefalteten Händen. ${ }^{4}$

Was Etty Hillesum hier als blosse Möglichkeit, niederkniend aus dem Tosen in die Ruhe zu kommen, erahnt, ohne es selbst zu können, wird im Laufe der Monate mehr und mehr stattfinden. So heisst es, kurz nachdem sie von ihrer Erzählung über das nicht niederkniende Mädchen gesprochen hatte, am 3. Dezember 1941: »Ich knie wieder auf der rauen Kokosmatte", und diesmal verbindet sich damit auch gleich ein ausformuliertes Gebet: „O Herr, lass mich aufgehen in einem einzigen grossen und ungeteilten Gefühl. Lass mich die tausend kleinen alltäglichen Dinge mit Liebe verrichten, aber lass jede kleine Handlung immer mehr zu einer regelmässigen Haltung aus einem einzigen grossen, zentralen Gefühl der Bereitschaft und Liebe hervorgehen. $\|^{5}$ Obschon sie manchmal von einem Einüben spricht, ist es eigentlich kein Erlernen im strengen Sinne, denn sie kommt spontan in diese Position des Niederkniens, ohne es bewusst zu wollen. Das wird in einer Notiz vom 14. Dezember 1941 betont: "Gestern Abend, kurz bevor ich mich hinlegte, fand ich mich plötzlich auf den Knien mitten in diesem grossen Zimmer, zwischen den

4 Les écrits d'Etty Hillesum, 63 (Übersetzung P. Bü.).

5 Das denkende Herz der Baracke, 120. 
Metallstühlen, auf dem Teppich mit hellen Tönen. Einfach so, ohne es gewollt zu haben. Zum Boden geneigt durch einen Willen, der stärker ist als meiner. Und vor einiger Zeit sagte ich mir noch: >Ich übe das Niederknien ein. « ${ }^{6}$

An Karfreitag 1942 kann sie dann von diesem Niederknien als einer für sie natürlich gewordenen Bewegung sprechen, die sie in ihrer zutiefst leiblichen Dimension thematisiert. »Die natürliche Bewegung, niederzuknien, geht manchmal durch meinen ganzen Leib», und diese erste Formulierung wird gleich korrigiert, im Versuch, es noch besser zum Ausdruck zu bringen:

"Es ist als ob die Geste des Niederkniens in meinem ganzen Leib modelliert wäre, ich spüre sie manchmal in meinem ganzen Leib. Manchmal, in Momenten tiefer Dankbarkeit, kommt mir ein unwiderstehliches Bedürfnis niederzuknien, den Kopf tief nach unten gesenkt, das Gesicht in den Händen. Es ist eine in meinen Leib eingegossene Geste, und die manchmal verwirklicht werden will. Und ich erinnere mich an >das Mädchen, das nicht wusste, wie niederknien<, und an die Rauheit der Kokosmatte im Badezimmer. " ${ }^{7}$

Im Rückblick auf »das Mädchen, das nicht knien konnte« wird hier die Geste des Niederkniens als etwas wahrgenommen, das tief zum Leben gehört, hier als Moment der Dankbarkeit, aber an anderen Stellen auch in Momenten der Sorge, der Bedrängnis und der Not zum Vollzug kommend. Und zwar als eine leiblich erfahrene Wirklichkeit: eine in den Leib eingegossene, im Leib modellierte Geste, so dass dieses Niederknien als ein "unwiderstehliches Bedürfnis" erlebt wird. Das Beten scheint hier unlösbar mit der Verleiblichung des Niederkniens verbunden zu sein, und dass die raue Kokosmatte des Badezimmers immer wieder erwähnt wird, zeugt von dieserVerleiblichung, vielleicht mit einem leichten Zug zum Asketischen. Das Badezimmer oder die Kammer dienen hier dem Abgesondertsein, denn das Niederknien ist etwas sehr Intimes. In derselben Notiz heisst es deshalb: "Beim Schreiben darüber doch das Gefühl einer gewissen Hemmung, wie wenn man das Intimste des Intimsten ansprechen würde.Viel mehr Scheue und Zurückhaltung, als wenn ich über mein Liebesleben sprechen würde. Aber was könnte es Intimeres geben als das Verhältnis der Menschen zu Gott? « Deshalb macht sie sich dann auch gleich lustig über ein Treffen der OxfordBewegung: „Und deshalb auch eine gewisse Abneigung gegenüber

6 Les écrits d'Etty Hillesum, 265 (Übersetzung P. Bü.).

7 Ebd., 451 [3.4.1942] (Übersetzung P. Bü.). 
der Oxford-Versammlung vor einigen Tagen. Welcher Exhibitionismus! Wie wenn man mit Gott öffentlich Liebe machen würde! « ${ }^{8}$

Dass beim Niederknien der Kopf nach unten gesenkt wird, scheint nicht mit einer Demutsgeste verknüpft zu sein, obschon sie davon spricht, dass ein stärkerer Wille sie zum Boden neigt. Es bekommt eine andere symbolische Interpretation, mit Hilfe der Metapher des Brunnens: "In mir gibt es einen ganz tiefen Brunnen. Und darin ist Gott. Manchmal ist er für mich erreichbar. Aber oft liegen Steine und Geröll auf dem Brunnen und dann ist Gott begraben. Dann muss er wieder ausgegraben werden. $\|^{9}$ Dieses Bild eines Grabens in ihr, in dem Gott zu finden ist, in dem er immer wieder ausgegraben werden muss, führt Etty Hillesum gleich zur Unterscheidung zwischen zwei Arten, das Beten auszurichten:

"Ich stelle mir vor, dass es Menschen gibt, die beim Beten die Augen zum Himmel erheben. Sie suchen Gott ausserhalb ihrer selbst. Es gibt auch andere, die den Kopf senken und in den Händen verbergen, ich glaube, diese Menschen suchen Gott in sich selbst."

\section{»Hineinhorchen«}

Dass Etty Hillesum zur zweiten Gruppe gehört, kommt leiblich dadurch zum Ausdruck, dass das Senken des Hauptes und das Verhüllen des Gesichts in den Händen auch immer zur Geste des Niederkniens gehören. Zugleich zeigt sich in vielen Tagebuchnotizen, dass es in der Tat darum geht, Gott in sich selbst zu suchen, in sich selbst auf ihn zu hören, ja sogar ihm in sich selbst Raum zu gewähren. »Es ist das einzige, auf das es ankommt: ein Stück von dir in uns selbst zu retten, Gott. Und vielleicht können wir mithelfen, dich in den gequälten Herzen der anderen Menschen auferstehen zu lassen. ${ }^{10}$

Diese Ausrichtung nach innen hin wird später in den Tagebüchern als ein "In sich ruhen "beschrieben: "Und hiermit ist mein Lebensgefühl wohl am vollkommensten ausgedrückt: Ich ruhe in mir selbst. Und jenes Selbst, das Allertiefste und Allerreichste in mir, in dem ich ruhe, nenne ich >Gott. ॥ $^{11}$ Doch ist dieses »Ruhen» nicht einfach Inaktivität, denn bereits in derselben Tagebuchnotiz

Beide Zitate ebd. (Übersetzung P. Bü.).

9 Das denkende Herz der Baracke, 81 [26.8.1941].

${ }^{10}$ Ebd., 224 [12.7.1942].

${ }^{11}$ Ebd., 262 [17.9.1942]. 
wechselt die Autorin von der Thematik des Ruhens zur Bewegung des Hineinhorchens. »Hineinhorchen<, dafür möchte ich einen guten holländischen Ausdruck finden. Eigentlich ist mein Leben ein unablässiges >Hineinhorchen in mich selbst, in andere und in Gott." Auch hier wird die Grundperspektive gleich umgekehrt, als ein Hineinhorchen Gottes: „Und wenn ich sage, dass ich shineinhorche<, dann ist es eigentlich Gott, der in mich shineinhorcht «." Und diese Umkehr wird folgendermassen korrigiert: "Das Wesentlichste und Tiefste in mir, das auf das Wesentlichste und Tiefste in dem anderen horcht. Gott zu Gott. «12

Dass dieses Hineinhorchen ebenfalls mit der leiblichen Gebetshaltung des Niederkniens zu tun hat, zeigt folgende Notiz vom 31. Dezember 1941:»Und jetzt muss ich manchmal plötzlich niederknien, sogar in einer klaren Winternacht vor meinem Bett. Und in mich hineinhören. Mich leiten zu lassen, nicht von dem, was von aussen auf mich zukommt, sondern was innerlich in mir aufsteigt. Es ist erst ein Anfang. Ich weiss. Aber es ist kein zauderndes Beginnen mehr, es ist bereits gut fundiert. $\ll^{13}$

Im Mai 1942, als die Bedrohung durch die Judenverfolgung massiv zunimmt, betrachtet sie das Gebet als einen Schutz, eine Wand, eine Zelle, in der sie immer wieder ein Gesammeltsein erfährt, das ihr erlaubt, gestärkt wieder in die Welt hinauszugehen:

»Die Bedrohung von aussen wird ständig grösser, der Terror wächst mit jedem Tag. Ich ziehe das Gebet wie eine dunkle, schützende Wand um mich hoch, ziehe mich in das Gebet zurück wie eine Klosterzelle und trete dann wieder hinaus, 'gesammelter, stärker und wieder gefasst. [...] Die innere Konzentration errichtet hohe Mauern um mich, in denen ich zu mir selbst zurückfinde, mich aus allen Verstreutheiten wieder zu einem Ganzen zusammenfüge. Und ich könnte mir vorstellen, dass Zeiten kommen, in denen ich tage- und nächtelang auf den Knien bleiben werde, bis ich endlich fühle, dass mich wieder Mauern umgeben, in deren Schutz ich nicht an mir selbst verzweifle, mich nicht verliere und zugrunde gehe. ${ }^{14}$

${ }^{12}$ Ebd., 263 (alle Zitate auf dieser Seite).

13 Ebd., 133.

${ }^{14}$ Ebd., 154f [18.5.1942]. 


\section{»Das einzige menschenwürdige Verhalten«}

Mitte Juli 1942 bekam Etty Hillesum beim Judenrat in Amsterdam eine Anstellung für administrative Aufgaben. Sie wird es dort nicht lange aushalten, bis sie dann Ende Juli als Sozialhelferin ins Transitlager Westerbork kommt. Die zweiwöchige Anstellung an der "Kulturellen Abteilung" nimmt sie zwar als Gelegenheit wahr, die Menschen kennen zu lernen. Es ist aber ein ständiger Rummel, mit einer Riesenmenge von Menschen, die mit ihren jeweiligen Anliegen kommen und ihre Privilegien geltend machen: "Sie hassen, sie sind in Bezug auf ihre eigene Person optimistisch verblendet, sie intrigieren und verteidigen ehrgeizig ihre Pöstchen, das Ganze ist ein riesiger Saustall, und es gibt Augenblicke, in denen ich meinen Kopf mutlos auf die Schreibmaschine legen und sagen möchte: Ich kann das nicht mehr aushalten. ${ }^{15}$

In diesem Kontext wird ihr das Niederknien zu einer Art Protestzeichen gegen diesen "Saustall« des menschlichen Interessen- und Privilegienkampfes, der durch die deutsche Unterdrückung der jüdischen Bevölkerung gestiftet wird, zu einem Zeichen der Ergebenheit:

"Als ich heute durch den übervollen Korridor ging, verspürte ich plötzlich den Drang, dort auf dem Steinboden, inmitten all der Menschen niederzuknien. Das einzige menschenwürdige Verhalten, das uns in dieser Zeit noch geblieben ist: das Knien vor Gott. ${ }^{16}$

Diese Ergebenheit, die in diesem Niederknien zum Ausdruck kommt, die Bereitschaft, ein Massenschicksal auf sich zu nehmen, anstatt Privilegien herauszuholen, ist für sie nicht einfach eine Kapitulation. Immer wieder betont sie, dass sich damit auch eine ständige moralische Entrüstung verbindet. Deshalb kann sie die Aktivitäten, die sie zu vollbringen hat, sehr kritisch beurteilen. Und auch der Judenrat selbst, in seinem ambivalenten Mitmachen bei den Deportationen, wird scharf ins Gericht genommen: »Es ist wohl nie wiedergutzumachen, dass ein kleiner Teil der Juden mithilft, die überwiegende Mehrheit abzutransportieren. Die Geschichte wird später ihr Urteil darüber fällen. «17

Etty Hillesums Niederknien, das hier auch Züge eines politischen Engagements annimmt, hat sich nicht ritualisiert, denn es blieb ein

${ }^{15}$ Ebd., 240 [23.7.1942].

${ }^{16}$ Ebd.

${ }^{17}$ Ebd., 250 [28.7.1942]. 
spontan eintretendes Verhalten, über das sie selber öfters staunte. Und später tritt es manchmal auch aus der Zurückgezogenheit des Badezimmers oder der Kammer heraus: So etwa im Transitlager Westerbork, wo es in äusserst schwieriger Leidenssituation auch draussen, beim Stacheldraht, vor der weiten Heide, stattfinden kann. Mit Jopie, einem engen Freund unter den Mitarbeitern des Lagers, "unter dem grossen Sternenhimmel, auf der Heide sitzend «, geht es im Gespräch zunächst um Heimweh. »Ich habe kein Heimweh, ich bin doch zu Hause", sagt Jopie. Diesen Satz aufnehmend, schreibt sie: "Daraus habe ich damals so viel gelernt. Man ist zzu Hauser. Unter dem Himmel ist man zu Hause. Auf jedem Fleck der Erde ist man zu Hause, wenn man alles mit sich trägt. ${ }^{18}$ Das Gespräch wurde dann intimer, aber Etty Hillesum sagt, sie brauchte zwei Abende, um ihm ihr Intimstes erzählen zu können: »Ja, weisst du, ich bin nachts aus meiner Baracke heraus. Es war so schön, weisst du. [...] und dann bin ich da niedergekniet, auf dieser weiten Heide." Sie fügt hinzu: "Es verschlug ihm den Atem, er war still, schaute mich an und sagte: ,Wie schön bist du. « ${ }^{19}$

\section{Zum Abschluss}

Das an unserem Institut angesiedelte SNF-Forschungsprojekt zum Thema Gebet fragt danach, wie in diesem »verleiblichtes Verstehen" geschieht. Kniend, den Kopf tief nach unten gesenkt, das Gesicht in den Händen: In dieser leiblichen Haltung hat Etty Hillesum ihre immer prekärer werdende Situation und die in ihr enthaltenen Herausforderungen zu verstehen versucht. "Prekär» darf hier im wörtlichen Sinn verstanden werden: Dieses Adjektiv kommt vom lateinischen "precarius", das mit dem Substantiv "prex, precis" zu tun hat, das "Gebet, Bitte " heisst. Ein prekäres Wesen ist ein auf Gebet angewiesenes Wesen, das das Lebensnotwendige immer wieder erbeten muss.

Das gilt auch für Etty Hillesum, deren Tagebücher im Laufe der Monate immer mehr zu einem regelmässigen Gespräch mit Gott werden. Die Prekarität ihrer Existenz wird im Gebet Gott anvertraut, und das Niederknien wird zum leiblichen Ausdruck dieses Anvertrauens, das zur Quelle ihres Selbstverständnisses wird. Es schenkt

\footnotetext{
18 Ebd., 268.

${ }^{19}$ Ebd. Die Schlussbemerkung zu Jopies Reaktion wurde aus der französischen Ausgabe übersetzt; in der deutschen Ausgabe wird die Szene abgekürzt mit "und habe ihm von Gott erzählt»!
} 
Ruhen in sich selbst, Hineinhorchen, und deshalb auch Leben und Handeln in freier Verantwortung.

Wittgenstein fürchtete im Niederknien das Weichwerden. Etty Hillesum wurde im Niederknien immer mehr gestärkt. Sie hat sich dabei nicht aufgelöst, sondern vielmehr gesammelt, um sich wieder in die Welt zu begeben, um dort den Schwachen, Elenden beizustehen. Das prekäre Beten stiftet Kraft, den prekären Existenzen in der Not zu helfen.

— Dr. Pierre Bühler ist Professor für Systematische Theologie, insbes. Hermeneutik und Fundamentaltheologie und Leiter des Instituts für Hermeneutik und Religionsphilosophie an der Universität Zürich. 